\title{
Bandwidth Utilization Enhancement for MPPM Using Gradual Multi-Pulse Pulse Position Modulation
}

\author{
Hossam Selmy \\ Department of Electronics and \\ Communications Engineering \\ Egypt-Japan University of Science and \\ Technology (EJUST) \\ Alexandria 21934, Egypt \\ Email: hossamselmy@yahoo.com
}

\author{
Hossam M. H. Shalaby \\ Department of Electronics and \\ Communications Engineering \\ Egypt-Japan University of Science and \\ Technology (EJUST) \\ Alexandria 21934, Egypt \\ Email: shalaby@ieee.org
}

\author{
Zen Kawasaki \\ Department of Electronics and \\ Communications Engineering \\ Egypt-Japan University of Science and \\ Technology (EJUST) \\ Alexandria 21934, Egypt \\ Email: zen@ejust.edu.eg
}

\begin{abstract}
Gradual multi-pulse pulse-position modulation (Gradual MPPM) is proposed as a new modulation technique to improve both the performance and bandwidth utilization efficiency of conventional optical multi-pulse pulse-position modulation (MPPM) scheme. While in conventional MPPM scheme, a fixed number of optical pulses is transmitted in every signal block, variable numbers of pulses are transmitted in the proposed scheme. Information is represented by different combinations of the positions of these pulses. The transmission characteristics, bandwidth utilization, and power requirements for the proposed scheme are studied in this paper. Several performance measures are derived and compared to that of conventional MPPM scheme. Our results reveal that the proposed modulation scheme achieves higher transmission energy efficiency (in bits per photon) at the same symbol error rate (SER). In addition, at the same average power, the proposed gradual MPPM scheme achieves much lower levels of SERs than that of the ordinary MPPM scheme. Furthermore, the proposed scheme allows much more symbols to be transmitted per frame than that of the ordinary MPPM scheme with the same frame size. This clearly leads to significant enhancement in the achieved bandwidth utilization efficiency.
\end{abstract}

\section{INTRODUCTION}

One of the most popular modulation techniques that is used in optical direct-detection channels is the single pulse position modulation (PPM) [1,2]. The advantage of using PPM is to locate the transmitted power efficiently in order to reduce the symbol error rate. Although, increasing the number of frame slots reduces the symbol error rate, it further reduces the bandwidth utilization efficiency and increases the synchronization errors. To overcome these drawbacks, the multi-pulse pulse position modulation (MPPM) was proposed [3]. In MPPM instead of transmitting a single optical pulse per frame, several pulses are allowed for transmission in order to increase their combinations per frame. Thus, a noticeable enhancement in bandwidth-utilization efficiency is achieved. Several performance comparisons between conventional single pulse PPM and multi-pulse PPM were made based on different criteria and under various transmission conditions [3][6].
The results indicate that the portion of bandwidth reduction achieved by MPPM is a function in number of optical pulses per frame. Also, using this scheme, the transmission bandwidth could be reduced to about half that of conventional optical PPM at the same information rate.

However, at the same information rate and allowing different bandwidth utilizations, the PPM outperforms MPPM in terms of bit error rates. Clearly, this performance gap is reduced when large number of slots per frame is implemented. Thus, different modulation formats are preferable under different transmission constraints, such as peak transmission power, average transmission power or bandwidth utilization.

In this paper, we propose a new modulation scheme that improves both the symbol error rate and the bandwidth utilization efficiency for the ordinary MPPM. In the proposed modulation scheme, which is called gradual multi-pulse pulse position modulation (gradual n-pulse M-PPM), we try to increase the number of transmitted symbols per frame while maintain a reasonable small number of slots per frame.

The rest of the paper is organized as: In Section II, we develop a general system model for gradual n-pulse M-PPM scheme and its variants. Also, the differences between the proposed gradual scheme and the ordinary scheme are stated. In Section III, we present the maximum-likelihood decoding (optimal decoding) for the proposed scheme on direct detected optical signals with the presence of background radiations. In Section IV, we derive performance measures in terms of the symbol-error rate under both average transmission power constraint and peak transmission power constraint. Finally, the performance comparisons and numerical results are presented in Section V.

\section{Proposed System Model And Characteristics}

In ordinary single pulse PPM with frame size of $M$ slots, the transmitted frames contain only one optical pulse that results in transmission of $\log _{2} M$ bits per frame. On the other side, in 


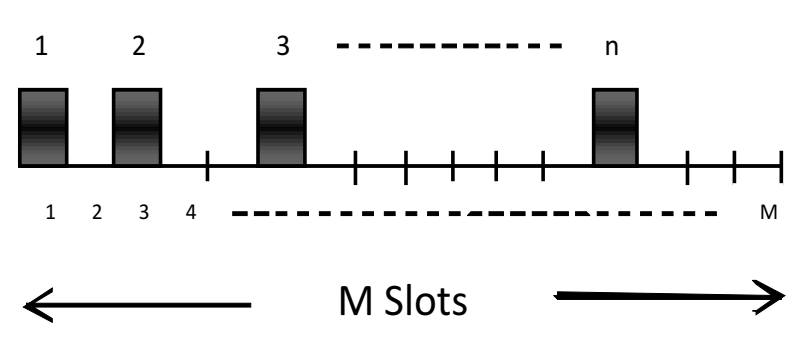

Fig. 1: Frame structure in ordinary MPPM
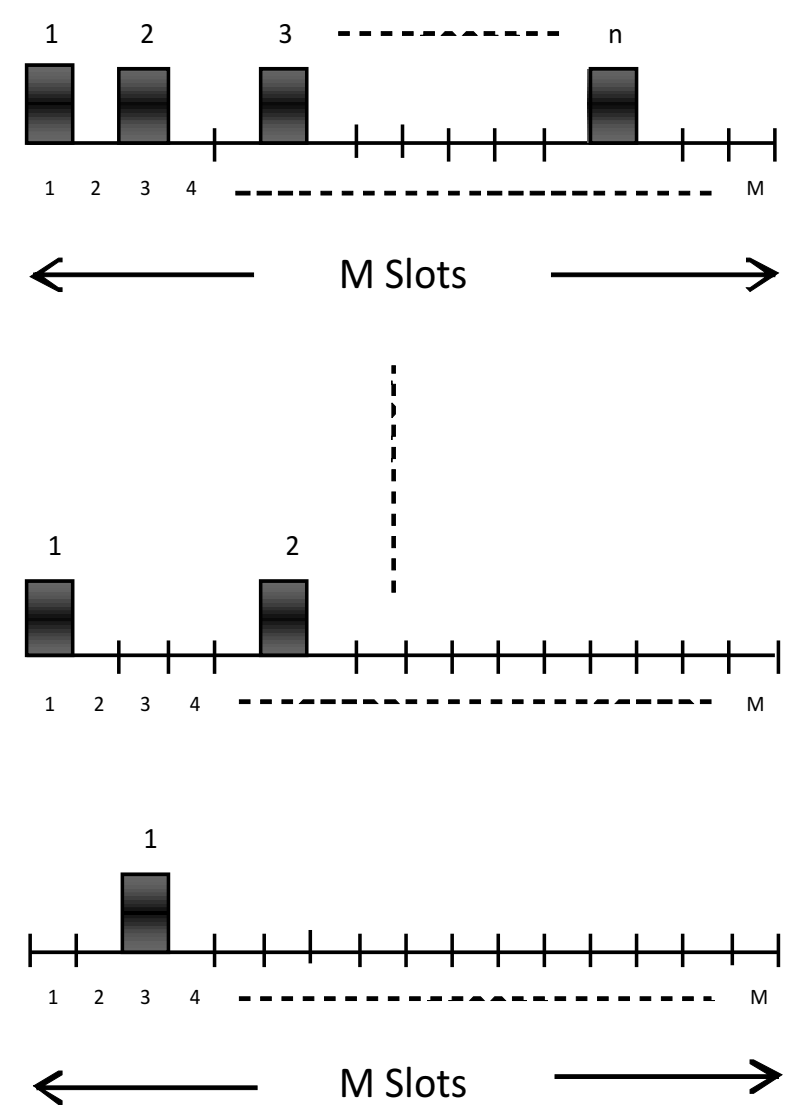

Fig. 2: Frame structure in gradual MPPM

the ordinary multi-pulse pulse position modulation (n-pulse MPPM), the transmitted frames contain $n$ optical pulses resulting in the transmission of $\log _{2}\left(\begin{array}{c}M \\ n\end{array}\right)$ bits per frame.

Clearly, in order to increase this number, the modulation constellations must be increased. To achieve that, we propose gradual multi-pulse pulse position modulation (gradual n-pulse M-PPM) scheme. In this scheme, instead of transmitting a fixed number of optical pulses ( $n$ pulses) per frame, we allow the transmission of one or more pulses (up to $n$ pulses) per frame. The resultant number of transmitted symbols per frame in this case is $\sum_{i=1}^{n}\left(\begin{array}{c}M \\ i\end{array}\right)$ symbols. Clearly, this number is much larger than the number of symbols available by ordinary MPPM schemes which is $\left(\begin{array}{c}M \\ n\end{array}\right)$ symbols. Thus, with the same frame size and same maximum number of transmitted optical pulses, the proposed gradual n-pulse M-PPM scheme achieves higher transmission capacity than the ordinary n-pulse M-PPM scheme. Furthermore, in the proposed modulation scheme, the maximum number of transmitted optical pulses per frame $n_{G}$ is allowed to increase largely up to the value of $M$ in contrast to the ordinary MPPM, where the value of $n_{M}$ is limited to $M / 2$. This valuable increasing in number of transmitted optical pulses per frame causes the bandwidth-utilization efficiency, achieved by the proposed scheme, to increase notably and reach near $100 \%$. Thus, another additional and important advantage rises for the proposed scheme.

The frame structure of both the ordinary MPPM and proposed gradual scheme are illustrated in Figs. 1 and 2, respectively. However, to clarify the idea of the proposed modulation scheme, we consider a numerical example in which the transmission frame consists of 8 slots. In ordinary single pulse PPM, the number of available symbols for transmission is 8 symbols producing a transmission of 3 bit per frame. For 2pulse 8-PPM scheme, there are $\left(\begin{array}{l}8 \\ 2\end{array}\right)=28$ symbols resulting in the transmission of 4.8 bits per frame. Using gradual 2pulse 8-PPM scheme, both single and double optical pulses are allowed for transmission resulting in a total of $8+28$ $=36$ symbols and a transmission of 5.16 bits per frame. An increase of about $10 \%$ is obtained in the information rate. The maximum transmission utilization achievable by MPPM scheme with frame size of 8 slots occurs when $n_{M}=4$. In this case, the number of available symbols is 70 symbols resulting in transmission of 6.12 bits per frame. For gradual 4-pulse 8 -PPM case, the number of available symbols is $8+28+56$ $+70=162$ symbols. Thus, achieving more than double the available symbols of ordinary 4-pulse 8-PPM.

Generally, in ordinary n-pulse M-PPM scheme, the number of transmitted bits per frame is $\log _{2}\left(\begin{array}{c}M \\ n_{M}\end{array}\right)$ (bits/frame) while in gradual n-pulse M-PPM scheme this number is $\log _{2} \sum_{i=1}^{n_{G}}\left(\begin{array}{c}M \\ i\end{array}\right)$. To cope for this, the bandwidth utilization efficiency for ordinary n-pulse M-PPM scheme is given by:

$$
U_{M}=\frac{\log _{2}\left(\begin{array}{c}
M \\
n_{M}
\end{array}\right)}{M}
$$

While the bandwidth utilization efficiency for gradual n-pulse M-PPM scheme is given by:

$$
U_{G}=\frac{\log _{2} \sum_{i=1}^{n_{G}}\left(\begin{array}{c}
M \\
i
\end{array}\right)}{M}
$$

Therefore, at the same values for $n_{M}$ and $n_{G}$, the information rates ratio is given by:

$$
\text { Information rates ratio }=\frac{\log _{2} \sum_{i=1}^{n}\left(\begin{array}{c}
M \\
i
\end{array}\right)}{\log _{2}\left(\begin{array}{c}
M \\
n
\end{array}\right)}
$$

As expected, the ratio is increased by increasing the maximum number of optical pulses per frame allowing more symbols to be available for transmission. Thus, the bandwidth utilization efficiency could be increased significantly using the proposed scheme which is highly intended to be used for applications that require efficient bandwidth utilizations. 


\section{Maximum LiKelihood Decoding}

Here, we aim obtaining the optimal decoding that minimizes the decoding error rate for the proposed gradual n-pulse $\mathrm{M}$ PPM scheme. To simplify the analysis, we consider a discrete memory-less optical channel with Poisson distribution for both signal and background optical radiations. The detected photoncounts associated with the slots of each received frame are summarized in the received M-component photon-count vector $\mathbf{k}=\left(k_{1}, k_{2}, k_{3}, . ., k_{n}, . ., k_{M}\right)$. Clearly, the probability of each count component is Poisson distributed with an average value of $K_{b}=\lambda_{b} * \tau$ ( $\lambda_{b}$ denotes the background intensity and $\tau$ is the slot time) when only background radiation is observed, and $\lambda_{z i} * \tau+K_{b}$ when both signal and background are present [7]. Here, the subscript $z$ refers to the transmitted symbol $Z$, whereas the subscript $i \in\{1,2, \ldots, M\}$ identifies the slot number within the count-vector and $\lambda_{z i}$ denotes the signal intensity in slot $i$ for transmitted symbol $Z$. Also, the value of $\lambda_{z i} * \tau$ is donated by $K_{s}$ if slot $i$ has an optical pulse.

Since, the received counts are independent on each other, the conditional probability of each count vector $\mathbf{k}$ given certain hypothesis symbol $X$ may be expressed as a product of individual count probabilities as.

$$
P(\mathbf{k} \mid X)=\prod_{i=1}^{M} \frac{\left(\lambda_{x i} \tau+K_{b}\right)^{k_{i}}}{k_{i} !} e^{-\left(\lambda_{x i} \tau+K_{b}\right)}
$$

The maximum likelihood decoding is to compute the conditional probabilities for all hypothesis symbols and to select the symbol that results in a maximum conditional probability for the received count vector. In order to get the symbol with the maximum conditional probability given the received count vector, we consider the following two cases:

Case A: Consider two symbols $X$ and $Y$ each contains $j$ ones, where $j \leq n$. The division of their maximum likelihood probabilities, given a count vector $\mathbf{k}$ is received, can be written as

$$
\begin{aligned}
\frac{P(\mathbf{k} \mid X)}{P(\mathbf{k} \mid Y)} & =\frac{\prod_{i=1}^{M} \frac{\left(\lambda_{x i} \tau+K_{b}\right)^{k_{i}}}{k_{i} !} e^{-\left(\lambda_{x i} \tau+K_{b}\right)}}{\prod_{i=1}^{M} \frac{\left(\lambda_{y i} \tau+K_{b}\right)^{k_{i}}}{k_{i} !} e^{-\left(\lambda_{y i} \tau+K_{b}\right)}} \\
& =\prod_{i=1}^{M}\left(\frac{\frac{\lambda_{x i} \tau}{K_{b}}+1}{\frac{\lambda_{y i} \tau}{K_{b}}+1}\right)^{k_{i}}
\end{aligned}
$$

Assuming that symbol $X$ has $j$ ones associated with the counts $k_{x_{1}}, k_{x_{2}}, . ., k_{x_{j}}$ whereas symbol $Y$ has $j$ ones associated with the counts $k_{y_{1}}, k_{y_{2}}, . ., k_{y_{j}}$. Also, ordering the received count vector $\mathbf{k}$ in a descending order to get the modified vector $\mathbf{k}^{*}=\left(k^{1}, k^{2}, . ., k^{n}, . ., k^{M}\right)$ then we have

$$
\frac{P(\mathbf{k} \mid X)}{P(\mathbf{k} \mid Y)}=\left(\frac{K_{s}}{K_{b}}+1\right)^{k_{x_{1}}+k_{x_{2}}+. .+k_{x_{j}}-k_{y_{1}}-k_{y_{2}}-. .-k_{y_{j}}}
$$

Clearly, if we select the symbol $X$ such that

$$
k_{x_{1}}+k_{x_{2}}+. .+k_{x_{j}}=k^{1}+k^{2}+. .+k^{j}
$$

Then $P(\mathbf{k} \mid X)>P(\mathbf{k} \mid Y)$ for any symbol $Y$ that contains $j$ ones. To summarize this point: For all symbols that have $j$ ones, the conditional probability given the received count vector will be maximized for the symbol that has its ones in the same positions with the positions of largest $j$ counts in the received count vector $\mathbf{k}$.

Case B: Consider two symbols $X$ and $Y$ which differ only in slot $l$, where $X$ symbol has one and $Y$ symbol has zero. Thus we have

$$
\begin{aligned}
\frac{P(k \mid X)}{P(k \mid Y)} & =\frac{\prod_{i=1}^{M} \frac{\left(\lambda_{x i} \tau+K_{b}\right)^{k_{i}}}{k_{i} !} e^{-\left(\lambda_{x i} \tau+K_{b}\right)}}{\prod_{i=1}^{M} \frac{\left(\lambda_{y i} \tau+K_{b}\right)^{k_{i}}}{k_{i} !} e^{-\left(\lambda_{y i} \tau+K_{b}\right)}} \\
& =\left(1+\frac{K_{s}}{K_{b}}\right)^{k_{l}} * e^{-K_{s}}
\end{aligned}
$$

Let us define the threshold $T h$ as

$$
T h=\frac{K_{s}}{\ln \left(1+\frac{K_{s}}{K_{b}}\right)}
$$

Then, $P(\mathbf{k} \mid X)>P(\mathbf{k} \mid Y)$ if $k_{l}>T h$. Clearly, from the mentioned two cases we could set the following optimal decoding (Maximum Likelihood Decoding) algorithm for the gradual MPPM scheme.

\section{Algorithm Steps}

1) Sort the received photo-counts in a descending order.

2) Decode the first maximum photo-count as one.

3) For $i=2$ to $n$

If (next maximum $\geq T h$ )

Then: Decode it as one

Else: Decode it and the remaining counts as zeros and end the algorithm.

4) Decode the remaining $M-n$ slots as zeros.

\section{Performance Evaluation}

In this section we aim at evaluating the error rate for gradual n-pulse M-PPM. We recall the calculation of SER for the ordinary n-pulse M-PPM as stated in [8]. For memory-less channel, without loss of generality, we could assume that the signal slots of the transmitted symbol occupy the first $n$ slots of the frame. Then, the probability to decode the received count vector $\mathbf{k}$ correctly is the probability that $\min \left(k_{1}, k_{2}, . ., k_{n}\right) \geq$ $\max \left(k_{n+1}, . ., k_{M}\right)$. If the equality holds, the decoder chooses randomly among multiple decoding decisions and only one of them is correct. To compute this probability, let $p_{0}\left(k_{i}\right)$ and $p_{1}\left(k_{i}\right)$ denote the count probabilities of slot $i$ in case of non-signal and signal slot, respectively. Also, let $P_{1}\left(k_{i}\right)$ and $P_{1}\left(k_{i}\right)$ denote their cumulative distributions. Furthermore, assume $k_{\max }=\max \left(k_{n+1}, . ., k_{M}\right)$ with $l$ repetitions in the $M-n$ non-signal slots and $m$ repetitions in the $n$ signal slots i.e. $1 \leq l \leq M-n, 0 \leq m \leq n$. Clearly, for any values of $l$ and $m$, there are $\left(\begin{array}{c}l+m \\ m\end{array}\right)=\frac{1}{I(l, m)}$ different decoding decisions with only one of them is correct. Thus, symbol error rate for $\mathrm{n}$-pulse M-PPM is given by 


$$
\begin{aligned}
S E R= & 1-\sum_{k_{\max =0}}^{\infty} \sum_{l=1}^{M-n} \sum_{m=0}^{n} I(l, m)\left(\begin{array}{c}
M-n \\
l
\end{array}\right) p_{0}\left(k_{\max }\right)^{l} \\
& * P_{0}\left(k_{\max }-1\right)^{M-n-l}\left(\begin{array}{c}
n \\
m
\end{array}\right) p_{1}\left(k_{\max }\right)^{m} \\
& *\left(1-P_{1}\left(k_{\max }\right)\right)^{n-m}
\end{aligned}
$$

Next we evaluate the SER for the gradual n-pulse M-PPM for $n>1$. However, the case $n=1$ (i.e., ordinary PPM) cannot be considered as a special case of gradual MPPM and the following analysis cannot be applied, instead it may be considered as a special case of n-pulse M-PPM with $n=1$. Here, we first evaluate the probability to receive the transmitted symbols correctly which is denoted by $p(c)$ and then we evaluate the required SER. However, in our evaluation of $p(c)$, we must consider the following three different cases of the transmitted symbols:

Case A: In this case, we consider the transmitted symbols that contain only one signal slot and the remaining $M-1$ slots are non-signal slots. Considering one of such symbols which has its signal pulse in the first slot, the probability to receive this symbol correctly is the probability that $k_{1} \geq \max \left(k_{2}, k_{3}, . ., k_{M}\right)$ and $\max \left(k_{2}, k_{3}, . ., k_{M}\right)<T h$. If the first equality holds i.e $k_{1}=\max \left(k_{2}, k_{3}, . ., k_{M}\right)$, then there are multiple decoding decisions and only one of them is correct. To count this, let $k_{\max }=\max \left(k_{2}, k_{3}, . ., k_{M}\right)$ and let $l$ denote the number of non-signal slots that have this count value. Here, the decoder chooses randomly one slot out of $l+1$ slots to be decoded as one and other as zeros. Thus, the probability to make correct decision is $I(l, 1)=\frac{1}{l+1}$. Also, we have $\left(\begin{array}{c}M-1 \\ l\end{array}\right)$ similar ways corresponding to the number of possible combinations for the remaining $M-1$ slots. Summing all the combinations for values of $l$ from 1 to $M-1$ and considering the two events $m=0$ when $k_{1}>k_{\max }$ and $m=1$ when $k_{1}=k_{\max }$, we obtain the total probability to receive this symbol correctly as:

$$
\begin{aligned}
p(c)_{A}= & \sum_{k_{\max }=0}^{T h-1} \sum_{l=1}^{M-1} \sum_{m=0}^{1} I(l, m)\left(\begin{array}{c}
M-1 \\
l
\end{array}\right) * p_{0}\left(k_{\max }\right)^{l} \\
& * P_{0}\left(k_{\max }-1\right)^{M-1-l} p_{1}\left(k_{\max }\right)^{m} \\
& *\left(1-P_{1}\left(k_{\max }\right)\right)^{1-m}
\end{aligned}
$$

Case B: In this case, we compute the probability of correct transmission for symbols that contain a number of signal slots $i$ that is greater than one and less than $n(1<i<n)$. Without loss of generality, we consider one of such symbols, which has its pulses in the first $i$ slots and the remaining $M-i$ slots are non-signal slots. The decoding decision is made correctly only if $\left(k_{1}, k_{2}, . ., k_{i} \geq T h>k_{i+1}, . ., k_{M}\right)$. Therefore, the probability to decode this symbol correctly is given by

$$
p(c)_{B i}=\left[1-P_{1}(T h-1)\right]^{i} *\left[P_{0}(T h-1)\right]^{M-i}
$$

Case $C$ : In this case, we compute the probability of correct transmission for the symbols that contain $n$ signal slots. Considering one of such symbols which has its pulses in the first $n$ slots and the remaining $M-n$ slots are nonsignal slots, the decoding decision is made correctly for this symbol only when $\min \left(k_{1}, k_{2}, . ., k_{n}\right) \geq \max \left(k_{n+1}, . ., k_{M}\right)$ and $\min \left(k_{1}, k_{2}, . ., k_{n}\right) \geq T h$. However, if the second equality holds, multiple decisions could be made and only one of them is correct. To consider these possibilities, let $k_{\text {min }}=$ $\min \left(k_{1}, k_{2}, . ., k_{n}\right)$. Also, let $l$ and $m$ denote the number of signal slots and non-signal slots that have this count value, respectively i.e., $0 \leq l \leq M-n$ and $1 \leq m \leq n$. Here, the decoder chooses randomly $m$ slots out of $l+m$ possible slots with probability of correct selection equals to $I(l, m)=\frac{1}{\left(\begin{array}{c}l+m \\ l\end{array}\right)}$. Also, there are $\left(\begin{array}{c}n \\ m\end{array}\right) *\left(\begin{array}{c}M-n \\ l\end{array}\right)$ different ways corresponding to the number of possible combinations for each value of $l$ and $m$. Summing all the combinations for values of $m$ from 1 to $M-n$ and values of $l$ from 1 to $n$, we obtain the probability of correction decoding for this symbol as:

$$
\begin{aligned}
p(c)_{c}= & \sum_{k_{m i n}=T h}^{\infty} \sum_{l=0}^{M-n} \sum_{m=1}^{n} I(l, m)\left(\begin{array}{c}
M-n \\
l
\end{array}\right) \\
& * P_{0}\left(k_{\text {min }}-1\right)^{M-n-l}\left(\begin{array}{c}
n \\
m
\end{array}\right) p_{1}\left(k_{m i n}\right)^{m} \\
& *\left(1-P_{1}\left(k_{\text {min }}\right)\right)^{n-m}
\end{aligned}
$$

Finally, the total probability of correct symbol transmission for gradual n-pulse M-PPM can be evaluated as the average probability of the correct decoding over all the transmitted symbols and thus the SER is given by:

$$
\begin{aligned}
S E R= & 1-p(c) \\
= & 1-\frac{1}{\sum_{j=1}^{n}\left(\begin{array}{c}
M \\
j
\end{array}\right)} *\left[\left(\begin{array}{c}
M \\
1
\end{array}\right) * p(c)_{A}\right. \\
& \left.+\sum_{i=2}^{n-1}\left(\begin{array}{c}
M \\
i
\end{array}\right) * p(c)_{B i}+\left(\begin{array}{c}
M \\
n
\end{array}\right) * p(c)_{C}\right]
\end{aligned}
$$

\section{NUMERICAL RESUltS}

In this section we compare the performance of the proposed gradual n-pulse M-PPM to ordinary n-pulse M-PPM in terms of the average symbol error rate, average received power and peak received power. To clarify the comparison, we use same frame size $\mathbf{M}$ and same slot duration $\tau$ for both schemes resulting in same frame rate. The simulations are 


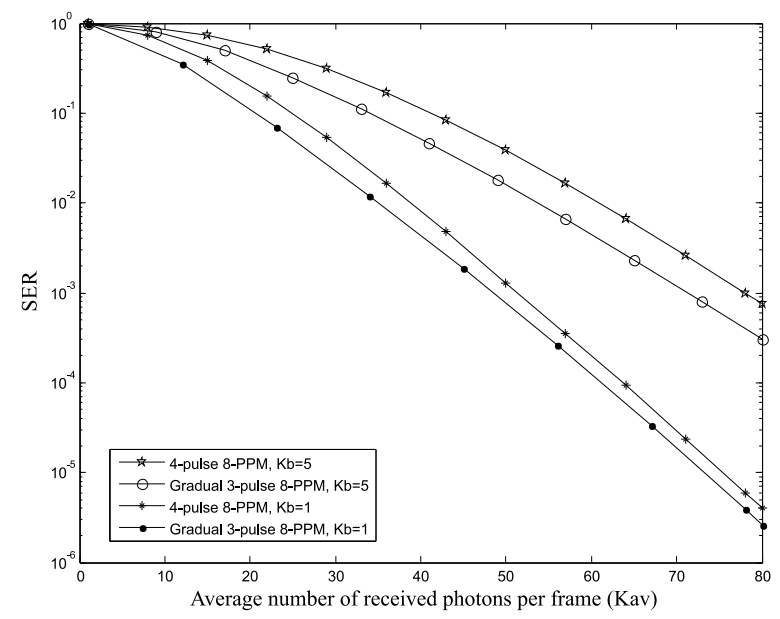

Fig. 3: Symbol error rate at $M=8$

carried for two values of the frame size, namely $M=8$ and $M=16$. For the case $M=8$, in order to achieve near the same number of transmission symbols for both schemes, the compared schemes are chosen to be 4-pulse 8-PPM (with number of signal pulses per frame $n_{M}=4$ ) and gradual 3-pulse 8-PPM (with maximum number of signal slots per frame $n_{G}=3$ ). In this selection the number of available symbols in 4-pulse 8-PPM scheme is 70 symbols while in gradual 3-pulse 8-PPM scheme, the number of the available symbols for transmission is 92 symbols. Clearly, gradual 3pulse 8-PPM achieves much more transmission capacity than 4-pulse 8-PPM which represents another advantage in their comparison. For the case, $M=16$ we choose 8-pulse 16-PPM and gradual 6-pulse 16-PPM schemes to compare. Clearly, the numbers of symbols available for transmission per frame for these modulations are 12870 and 14892 symbols, respectively, which add an extra advantage of the gradual scheme.

Furthermore, the background radiations (noise photons) are considered in the carried evaluations by the mean of average number of the received background photons per slot $K_{b}$. The simulations are performed at two noise levels which are $K_{b}=$ 1 and $K_{b}=5$.

On the other side, in terms of the received optical power, the comparisons are performed under average power constraint. This constraint is usually imposed in the case where the transmitter has limited power resources. That is, the transmitter has a bound on the number of emitted photons per transmitting frame. These cases rise for FSO systems working on solar energy and space optical communications between spacecrafts. Clearly, the modulation scheme that achieves higher performance at the same average energy level is highly desired in this case.

To minimize the compared parameters, we fix the frame size for both schemes.Thus, the average power comparison can be replaced by the average number of received photons per frame $K_{a v}$. The relation between the average number of the received photons per frame $K_{a v}$ and the signal slot photons $K_{s}$ are different for the two schemes.

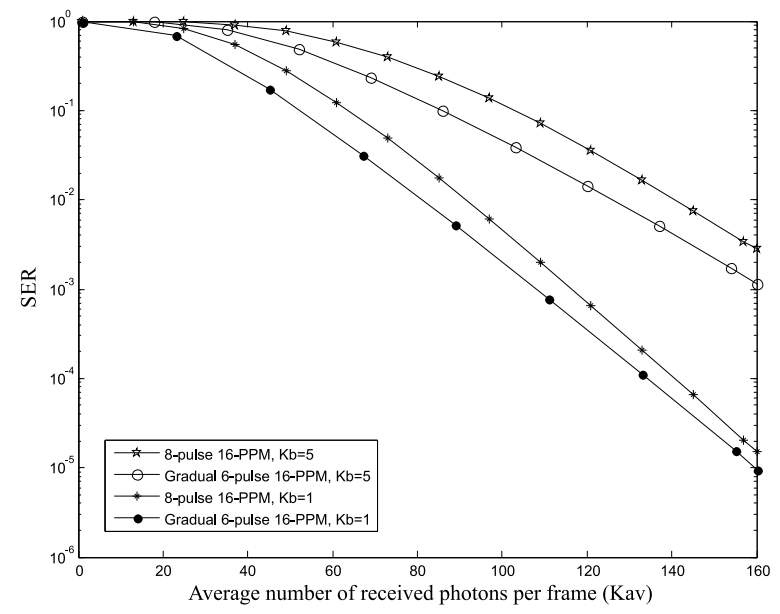

Fig. 4: Symbol error rate at $M=16$

For n-pulse M-PPM the average number of the received photons per symbol $K_{a v M}$ is given by

$$
K_{a v M}=K_{s M} * n_{M}
$$

For gradual n-pulse M-PPM the average number of the received photons $K_{a v G}$ is given by

$$
K_{a v G}=\frac{K_{s G} * \sum_{i=1}^{n_{G}} i *\left(\begin{array}{c}
M \\
i
\end{array}\right)}{\sum_{i=1}^{n_{G}}\left(\begin{array}{c}
M \\
i
\end{array}\right)}
$$

For equal average power constraint we have $K_{a v M}=$ $K_{a v G}$. Figures 3 and 4 show the first group of comparison, where we evaluate the performance of the proposed gradual n-pulse M-PPM scheme with the performance achieved by n-pulse M-PPM scheme in terms of received symbol error rate versus average number of the received photons per frame as given by (10) and (14). The results for the case $M=8$ are shown in fig. 3, which carry the comparison between two specific schemes: 4-pulse 8-PPM and gradual 3-pulse 8PPM. Obviously, as mentioned before, the gradual scheme achieves higher bandwidth utilization than that of the ordinary one. Figure 3 indicates the out-performance of the proposed schemes in achieving less SER at different values of average received photons per frame $K_{a v}$.

The comparisons are carried at two values of the background noise $K_{b}=1$ and $K_{b}=5$. Also, the figure indicates better performance of the proposed scheme at larger background noise. Specifically, at $K_{a v}=40$ photons the gradual n-pulse M-PPM achieves $6 \mathrm{~dB}$ reduction in SER at $K_{b}=1$ and a reduction of $8 \mathrm{~dB}$ at $K_{b}=5$. This large reduction in SER at higher $K_{b}$ levels makes the gradual scheme noise robust. For the case $M=16$ as indicated in Fig. 4, the comparison is carried between two modulation schemes which are 8-pulse 16-PPM and gradual 6-pulse 16-PPM. Again, this selection achieves nearly same bandwidth utilization for both schemes with an advantage to the gradual one. The figure emphasizes the superior performance of the proposed gradual scheme over the ordinary one. Furthermore, this performance gab is increased by increasing the level of background noise. 


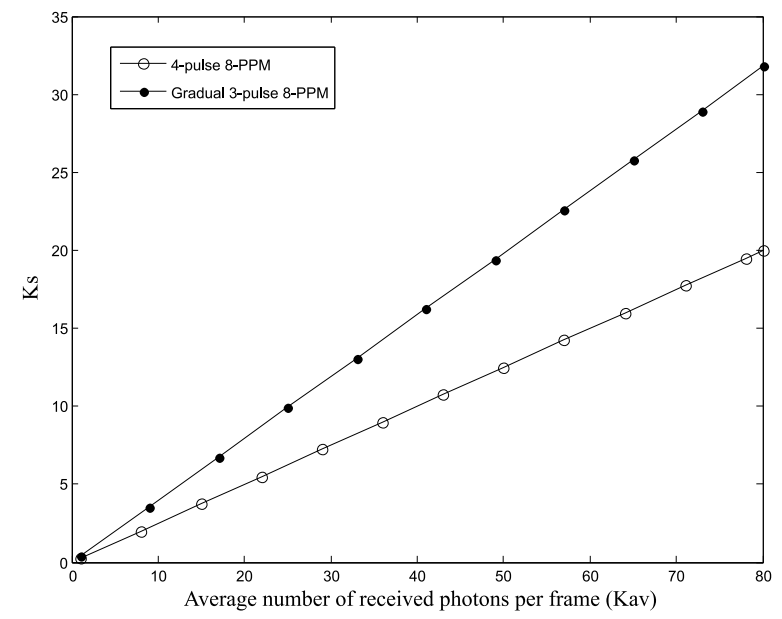

Fig. 5: $K_{s}$ at $M=8$

This could be interpreted as: at the same average number of received photons per frame $K_{a v}$, the pulse power in gradual scheme is much higher than pulse power in ordinary scheme which in turn causes the gradual scheme to perform much better in noisy channels.

The analysis of the achieved symbol error rate performance is explained further by the second group of figures, Figs. 5 and 6 . These figures indicate the effect of the average number of received photon per frame $K_{a v}$ (which represents a metric for the average power) on the number of received photons in signal slot $K_{s}$ (which represents a metric for the peak power). Obviously, in the gradual schemes, the average number of signal slots per frame is much less than the number of signal slots per frame in ordinary MPPM schemes with the same frame sizes. This fact along with transmitting the same average number of photons per frame $\left(K_{a v}\right)$ introduce a noticeable increase in the number of received photons per signal slot $\left(K_{s}\right)$ for the gradual schemes than that of the ordinary ones. Consequently, with the same background noise levels, i.e., same values of $K_{b}$, the detection of the gradual schemes symbols is less erroneous than that of the ordinary MPPM schemes. However, this performance enhancement comes with the price of noticeable increasing in peak power levels, which may not be desirable for both transmitter amplifier saturation and safety regulations.

Numerically, as indicated in Figs. 5 and 6, for the same average received power, the gradual n-pulse M-PPM schemes cause the number of received photons per signal slot $K_{s}$ and consequently the peak power to increase linearly with about one and half the increasing rate of the ordinary schemes. However, this increasing rate may limit the performance achieved by the gradual schemes to values lower than that achieved by the ordinary schemes in case the same maximum peak power level is used.

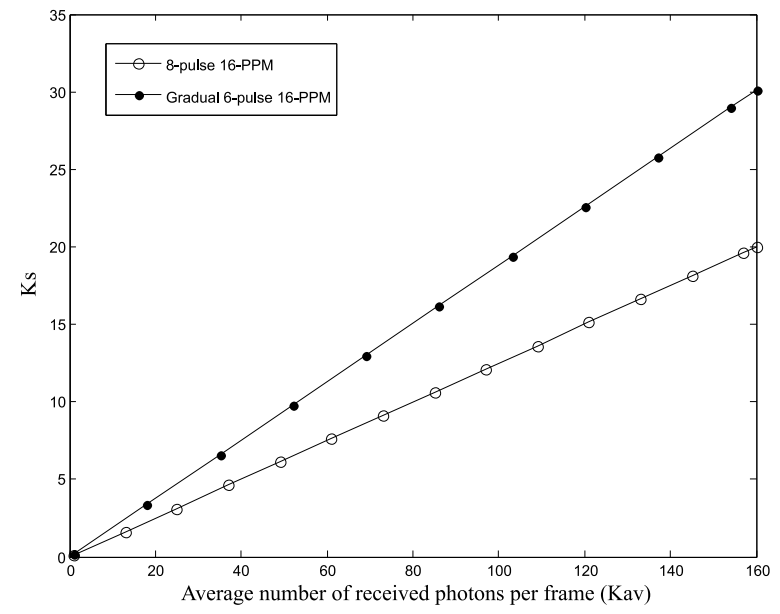

Fig. 6: $K_{s}$ at $M=16$

\section{Conclusion}

A new modulation scheme, gradual n-pulse M-PPM, of the family of pulse position modulations is proposed for optical communications. The proposed scheme achieves much higher bandwidth utilization efficiency than that of the ordinary MPPM scheme. On a discrete memory-less channel, the maximum likelihood decoding criteria for the proposed scheme is derived resulting in a simple and fast decoding algorithm. The performance measure of the proposed scheme in terms of exact symbol error rate is obtained. Intensive numerical evaluations are performed to compare the performance achieved by the proposed gradual scheme to the performance of ordinary MPPM scheme. The comparisons are carried under average power constraint at nearly the same transmission rates. The results indicate the superior performance of the proposed gradual scheme under that constraint.

\section{REFERENCES}

[1] J. Singh and V. K. Jain, "Performance Analysis of BPPM and M-ary PPM Optical Communication Systems in Atmospheric Turbulence," IETE Technical Review, Vol.25, Issue 4, July-Aug 2008.

[2] M. Simon and V. Vilnrotter, "Performance Analysis and Tradeoff for Dual-Pulse PPM on Optical Communications Channels with Direct Detection," IEEE Trans. Commun., vol. 52, no. 11, pp. 1969-1979, November 2004.

[3] H. Sugiyama and K. Nosu, "MPPM: A Method for Improving the Band Utilization Efficiency in Optical PPM," Journal of Lightwave Technology, vol. 7, no. 3, pp. 465-471, March 1989.

[4] H. Park, "Performance bound on multiple-pulse position modulation," Optical Review, vol. 10, no. 3, pp. 131-132, May 2003.

[5] M. F. Barsoum, B. Moision, M. Fitz, D. Divsalar and J. Hamkins, "Iterative coded pulse-position-modulation for deep-space optical communications," Information Theory Workshop, pp. 66-71, Sept. 2007.

[6] K. Sato, T. Ohtsuki, and I. Sasase, "Performance of coded multipulse PPM with imperfect slot synchronization in optical direct-detection channel," in Proc. IEEE International Communications Conference (ICC), vol. 1, 2004, pp. 121-125.

[7] Robert M. Gagliardi and Sherman Karp, Optical Communications. John Wiley \& sons Inc., Second Edition, 1995.

[8] J. Hamkins and B. Moision, "Multipulse PPM on Memoryless Channels," in International Symposium on Information Theory (ISIT), Chicago, Illinois, p. 336, June 2004. 